\title{
FFAGS FOR RAPID ACCELERATION
}

\author{
C. Johnstone ${ }^{\mathrm{a}, 1}$

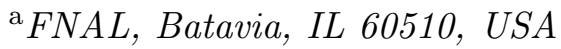 \\ S. Koscielniak ${ }^{\mathrm{b}, 2}$ \\ b TRIUMF, Vancouver, B.C., V6T 2A3 Canada
}

\begin{abstract}
When large transverse and longitudinal emittances are to be transported through a circular machine, extremely rapid acceleration holds the advantage that the beam becomes immune to nonlinear resonances because there is insufficient time for amplitudes to build up. Uncooled muon beams exhibit large emittances and require fast acceleration to avoid decay losses and would benefit from this style of acceleration. The approach here employs a fixed-field alternating gradient or FFAG magnet structure and a fixed frequency acceleration system. Acceptance is enhanced by the use only of linear lattice elements, and fixed-frequency rf enables the use of cavities with large shunt resistance and quality factor.
\end{abstract}

Key words:

PACS:

\section{Introduction}

Acceleration of large emittance beams, particularly those with simultaneously large transverse and longitudinal emittances, present a challenging new direction in accelerator design. Conventional accelerators such as synchrotrons or linacs cannot support acceleration of ultra-large emittances for either case.

Email addresses: cjj@fnal.gov (C. Johnstone), shane@triumf.ca (S.

Koscielniak).

1 Work supported by the Universities Research Association, Inc., under contract DE-AC02-76CH03000

2 TRIUMF receives funding via a contribution agreement through the National Research Council of Canada. 
Scaling FFAG accelerators, such as the radial or spiral sector[1], display an almost unlimited momentum acceptance, but transverse acceptance remains restricted. The approach described here is development of a nonscaling FFAG[2] wherein the ideal optics demonstrate strong linearity; that is, the acceptance is not sensitive to energy or amplitude. The discerning feature of the nonscaling version, in keeping with the condition of linearity, is that the optics are not held constant, as in the scaling machine, but change slowly with momentum.

Linearity in the base optics, however, does not guarantee beam survival. In spite of the use of strictly linear elements, sources of nonlinearity exist due to large-angle or kinematical corrections and unavoidable magnetic field errors. Often resonance behavior will be excited, depending on the timescale of the acceleration. However, the inclusion of slowly-changing optics in combination with rapid acceleration suppresses discrete resonances and nonlinear effects, thereby minimizing beam blowup and corresponding beam loss. Rapid acceleration has further application when the particle beam suffers from decay losses.

A signature of fixed field acceleration is that orbit length unavoidably changes with energy; it can be substantial and can result in a significant phase-slip relative to the accelerating waveform[3][2]. For relativistic beams and rapid acceleration, this poses a nonstandard problem which must be addressed by the rf system. Recent workshops have focussed on the phasing problems of FFAGs and a number of solutions are being advanced[11]. These include: (i) momentum-dependent chicanes[12] to correct pathlength differences, (ii) broad-band RF that can be phased quickly, or (iii) a frequency low enough (25 MHz, for example), to make the phase errors ineffectual. Outside of the obvious solutions of broadband rf, or very low frequency rf, this paper outlines alternative approaches using high-Q, high-frequency rf systems. This paper reports significant progress on both a lattice and rf acceleration system for a high-energy FFAG in the context of rapid acceleration.

\subsection{Application, nonscaling FFAG}

A strong example of the application of a nonscaling FFAG is for intense muon sources; e.g. a Muon Collider[4] or a Neutrino Factory[5][6] where acceleration must occur rapidly to avoid heavy decay losses. Linear acceleration is the most efficient, but above one $\mathrm{GeV}$ it becomes prohibitively expensive. Conventional synchrotrons cannot be used because normal conducting magnets cannot readily cycle in the ramping time required by muon decay. The current baseline approach employs recirculating linacs (RLAs) with separate, fixed-field arcs for each acceleration turn[4][7]. However, the RLA schemes only support 4 acceleration turns due to complications associated with passively switching a 
large-emittance beam between the arcs. A range of $2-4 \mathrm{GeV} /$ turn has so far been necessary to implement a RLA design for muon acceleration. (The circulation time is too rapid for a kicker-controlled switchyard.) Given the technical complexity, acceptance limitations, and expense of ultra-rapid cycling synchrotrons and recirculating linacs, the idea of using fixed-field, single-arc accelerators has been revisited in recent work and is the subject of this paper.

The exceptionally large acceptance of the nonscaling FFAG, both transversely and longitudinally, further impacts the degree of beam cooling required. For rapid acceleration, its acceptance appears limited only by the physical apertures of the components and can, in principle, accomodate less-cool beams than RLAs, or even scaling FFAGs. These studies of FFAG accelerators also represent an effort to reduce cost and promote an acceptance which is better matched to the performance of the ionisation cooling system and the storage ring.

Should give a few more citations?

\section{FFAG Lattices}

A circular accelerator system can be designed with magnetic fields that remain constant during acceleration by adopting an alternating gradient focussing lattice. The arcs of such machines, composed of large aperture magnets, can be designed to accommodate the large energy range in acceleration. The beam centroid orbit is not fixed as in a ramped machine, but rather moves across the magnet aperture during acceleration. Lattices have been developed which can contain an energy change of a factor of four[?]. The three types of alternating gradient structures used in FFAG lattice design are: (i) Traditional scaling FFAG; (ii) Triplet-based scaling FFAG; (iii) Nonscaling FFAG.

The traditional scaling FFAG is comprised of combined-function short FODO cells with edge focussing and magnetic fields which scale with momentum, leading to optics properties that are maintained constant as a function of momentum. Such FFAG rings were first designed and studied at MURA[8], and, more recently, for muon acceleration[2]. Keeping the orbit and optical properties consistent requires the magnetic field to scale with momentum, but achieving a large transverse dynamic aperture requires that $\mathrm{B}^{\prime}$ must be nearly constant. Unfortunately, this is incompatible with a large energy range, since it implies the horizontal spread of orbits becomes large. Curtailing the magnet aperture means the field must rise sharply with radius through addition of higher-order field terms. Degradation of dynamic aperture follows with a reduction of transverse acceptance. The triplet-based FFAG is a recent innovation developed for the KEK Proof of Principle machine[9]. Based on the 
scaled-field concept, but formed from a triplet quadrupole structure rather than a FODO one, it incorporates a significantly longer straight section in each cell facilitating injection, extraction, rf insertion, etc.

In the nonscaling FFAG, not only do the central orbits move across the aperture, but also the optics functions vary with the central momentum. When acceleration occurs so rapidly that the beam experiences only a few turns in the machine one does not have to avoid resonances or control lattice parameters as a function of momentum. Instead, one has the freedom to choose parameters optimal for the application such as minimizing circumference and requiring a large transverse dynamic aperture[2].

\subsection{Optics principles of a Nonscaling FFAG}

As mentioned earlier, when acceleration occurs rapidly resonances are not a consideration. The beam can be accelerated through an integer, or other resonance-driving "global" tunes if the tune remains constant for only a fraction of a turn. With a fast acceleration cycle (the rf systems are assumed to deliver on the order of $1 \mathrm{GeV}$ or more per turn), the lattice's optical parameters, such as betatron functions, may also change with momentum. Through the further choice of using only linear elements, an FFAG machine can be designed to support a large transverse dynamic aperture in addition to the large longitudinal one, again at the expense of maintaining constant optical properties. One then has the freedom to choose machine parameters which are optimal for acceleration; such as minimizing the circumference to limit intensity loss from decay and maximizing the transverse dynamic aperture. This approach has been termed a nonscaling FFAG accelerator.

Two steps are important in minimizing the machine circumference. First, the reverse bends required by the criterion to maintain constant optics can be eliminated yielding approximately a $20 \%$ decrease in total circumference. Then, also important, is to choose the magnet configuration in the basic FODO cell to provide the maximum net bend per cell for a given peak transverse excursion of the beam during acceleration. This is accomplished by favourably positioning the dipole bend field over the defocusing quadrupole element. The cells of the nonscaling ring then contain a horizontally focusing quadrupole followed by a vertically focusing, combined-function bending magnet. The allowed bend is further increased by the choice of focussing strength and cell length: the lower or injection momentum experiences a cell phase advance which approaches $\pi$ while the upper momentum approaches zero, depending sensitively on the choice of gradient and respective radial positions of the beam centroids (which determines total magnet aperture). The nonscaling approach

combined with this specific magnet layout yields the most compact design 
circumference of any lattice and can approach a factor of two less than that of a scaling lattice.

Relaxing the requirement for consistency in optical parameters at different energies means that the orbits no longer remain parallel. The peak of the orbit excursion always occurs at the center of the F quadrupole. Orbit excursions at low and high energy are almost inversions of one another. The need for a large transverse dynamic aperture is automatically satisfied in this design because only linear elements are used. Because of the short cell structure, variations in the maximum beta functions are not significant enough to instigate a serious beta wave during acceleration due to a transverse optics mismatch.

The example, which provides the focus of this paper, is a 6-20 GeV non-scaling FFAG cell optimized for ultra-rapid, stable acceleration, as is required for intense muon sources. The entire lattice is comprised solely from 314 simple arc FODO cells. (The periodicity is, therefore, 314). The rf system is assumed distributed over most of the ring with 3 cavities filling the $3 \mathrm{~m}$ straight in each half cell. (To keep field gradients to conventional values, $\approx 3 \mathrm{MV} / \mathrm{m}$, the rf needs to occupy about 300 of the cells, given a few number of acceleration turns.) The remaining empty cells are filled with the kickers necessary for injection and extraction, which require several cells to complete. The simplicity of lattice design; i.e. its unbroken periodic structure, is responsible for its stability over a large range in momentum despite changing optical conditions. The lattice components, parameters, betatron functions and peak orbit excursions are given in Table 1 and plotted in Figures 1 through 4. The central energy has been arbitrarily defined as the energy at which the beam centroid is centered in the focussing quadrupole (where $\mathrm{B}=0$ ). The plots of peak orbit excursions show the radial difference in the beam centroid positions at injection and extraction relative to the orbit described by the central energy.

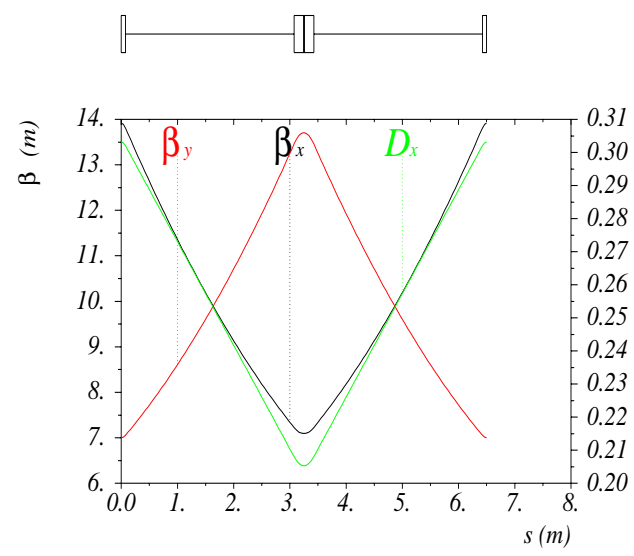

Fig. 1. Lattice functions at $16.5 \mathrm{GeV}$ for a $6-20 \mathrm{Fig}$. 2. Lattice functions at $6 \mathrm{GeV}$ for a $6-20$ GeV non-scaling FFAG.

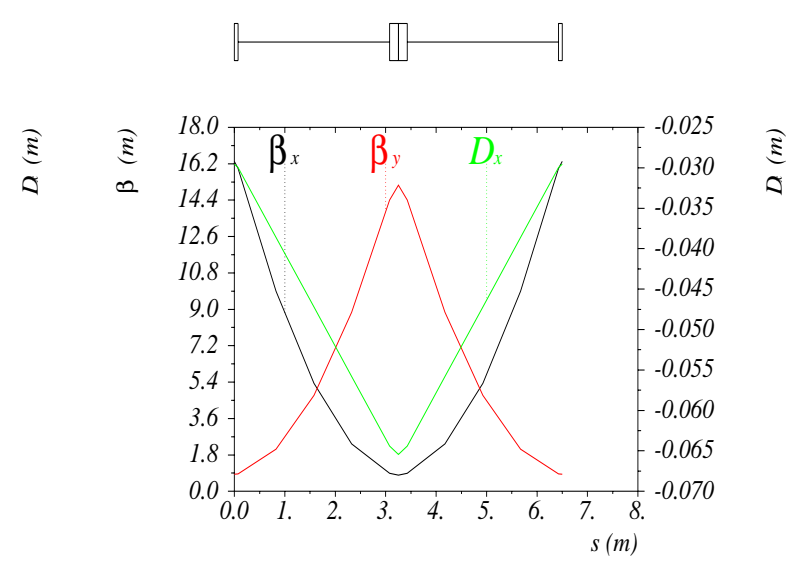

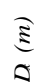

GeV non-scaling FFAG. 
Table 1

Parameters of a 6-20 GeV Nonscaling FFAG. Where two sets of values are given, they represent superconducting/normal conducting magnets, respectively.

General

$\begin{array}{cccc}\text { Central Energy } & 16.5 \mathrm{GeV} & \text { Circumference } & 2041 / 2355 \mathrm{~km} \\ \text { Rigidity } & 55 \mathrm{~T}-\mathrm{m} & \text { Poletip Field } & 6 \mathrm{~T} / 2 \mathrm{~T} \\ \text { Basic Cell } & & & \\ \text { Number } & 314 & \text { Length } & 6.5 / 7.5 \mathrm{~m} \\ \text { Bend/cell } & 0.02 \mathrm{rad} & \text { Quad Gradient } & 75.9 / 25.3 \mathrm{~T} / \mathrm{m} \\ \text { "F" length } & 0.15 / 0.45 \mathrm{~m} & \text { "F" strength } & 1.38 / 0.46 \mathrm{~m}^{-2} \\ \text { "D" length } & 0.35 / 1.05 \mathrm{~m} & \text { "D" strength } & 0.59 / 0.20 \mathrm{~m}^{-2}\end{array}$

\section{Cell Tunes}
$6 \mathrm{GeV}$
$0.45(162 \mathrm{deg})$
$20 \mathrm{GeV}$
$0.08(29 \mathrm{deg})$

$\underline{\text { Maximum }}$

$\underline{\text { Displacements }}$

$6 \mathrm{GeV}$

$-7.5 \mathrm{~cm}$
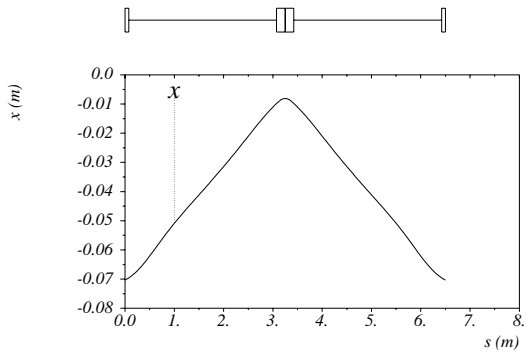

Fig. 3. 6-GeV orbit excursion for a 6-20 GeV non-scaling FFAG.
$20 \mathrm{GeV}$ $7.1 \mathrm{~cm}$

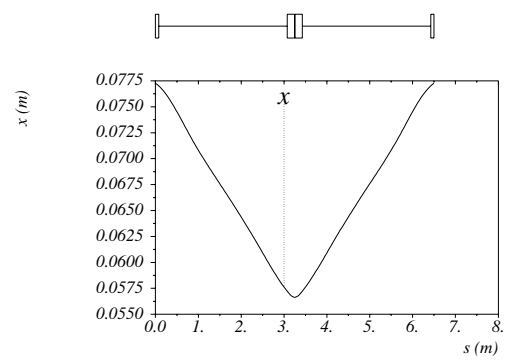

Fig. 4. 20-GeV orbit excursion for a 6-20 GeV non-scaling FFAG.

\subsection{Pathlength Dependencies in FFAGs}

A main drawback to FFAGs in both the scaling and the non-scaling versions is the large changes in pathlength as a function of energy. The pathlength dependence is clearly linear with momentum for radially-staggered, parallel orbits as in the radial-sector FFAG, but it is parabolic in nonscaling FFAGs. (The following discussion of scaling FFAGs refers to the radial-sector FFAG explicitly.) This comes about when the transverse excursion of orbits as a function of momentum (see Figure 3) is larger than the contribution from the longitudinal pathlength change. Nominally, for small momentum deviations from the central momentum, the fact that the lower momentum stays to the inside of the central orbit and the high momentum to the outside means a smaller total pathlength for low momentum and a larger one for higher momentum. The 
6-20 GeV Nonscaling FFAG

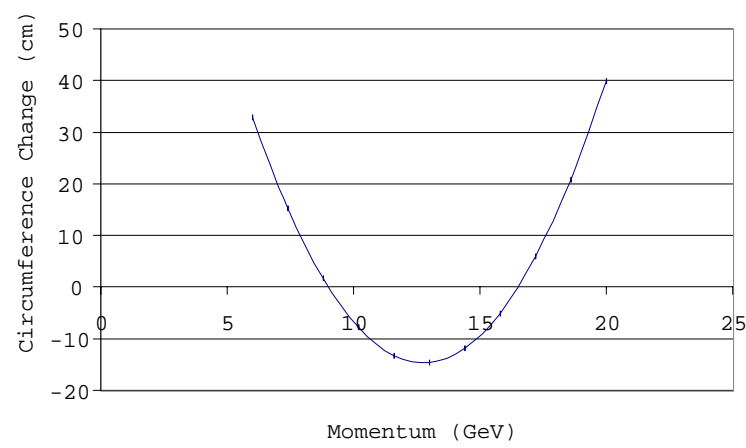

Fig. 5. Circumference change as a function of momentum

scaling FFAG follows this norm except the path length variations are large because of the large momentum acceptance. For large transverse apertures, and correspondingly large excursions across the magnet apertures, as is the case in the nonscaling FFAG, the transverse path changes overtake these longitudinal variations. Because of the large variations in their radial excursions, both high and low momentum have total pathlengths larger than the central orbit, giving a parabolic shape to the circumference change as a function of momentum (see Figure 5). The nonscaling FFAG passes through transition which lies at the minimum of the parabola and represents the midpoint of the acceleration range for the design lattices described here.

Pathlength and traversal time change with the reference energy. In a regime of slow acceleration this variation is distributed over many turns, but for rapid acceleration non-negligible changes occur from cell to cell. Of course, the cell traversal times must be synchronized with the waveforms in the RF cavities responsible for acceleration. This consideration leads to the following discussion of acceleration modes and the rf systems that are compatible with them.

\section{FFAG acceleration}

In a circular machine, particles make repeated passages through the same cavities; and so on every revolution of the machine the frequency and phasing of each cavity must be readjusted. To make this possible, the demanded phase change per cavity filling time should be small; and this leads to the condition $2 \pi(\Delta C / C)<1 / Q$ where $Q$ is the quality factor and $\Delta C / C$ is the relative fractional change in circumference per turn. If no attempt is made 
to adjust the cavity frequency or phase, then errors accumulate linearly with time and inversely as harmonic number. A fixed-field machine can be operated in several modes, classifiable in terms of the timescale for acceleration; for example, conventional and rapid, which range over tens of milliseconds and tens of microseconds, respectively. Between these extremes, elaborated below, lies moderate acceleration: theoretically, a few hundred acceleration turns (hundreds of microseconds) can be achieved in either a nonscaling or scaling FFAG.

\subsection{Conventional acceleration}

For a thousand, or more, turns acceleration, moderate-Q cavities $\left(10^{5}\right.$ or $\left.10^{6}\right)$ can be utilized and their phases made to track the changing orbit length, so as to make an approximation to conventional, synchronous phase acceleration. The longer timescale makes the nonscaling FFAG more sensitive to the optics design and the impact of resonances. Only the scaling FFAG, where tunes and betatron functions are held constant supports acceleration on a timescale comparable to a conventional synchrotron, albeit with an unavoidable degradation in the transverse acceptance.

\subsection{Rapid acceleration}

When acceleration is to be completed in a few turns, the large energy gain per turn forces one to consider on-crest acceleration as in a cyclotron. In this case, the rf is used almost entirely to provide acceleration. One may envisage use of low-Q $\left(\leq 10^{3}\right)$ cavities and on-crest operation with phase shifting of accelerating stations to make up for non-isochronous behaviour of orbits in the FFAG. Even when the phases of the cavities are readjusted, this regime can be sustained only for a few tens of turns, due to the near absence of phase focusing.

A more affordable approach for rapid acceleration employs high-Q $\left(\geq 10^{6}\right)$ and high frequency $(\geq 100 \mathrm{MHz}$ ). Because high-Q cavities cannot be rephased on a rapid acceleration timescale (microsecond), the phase relation of the beam to the acceleration waveform is completely determined by the changes in the orbital pathlength during acceleration, and these changes are substantial at high cavity frequency. (For the example in this paper, the total pathlength changes by $\approx 50 \mathrm{~cm}$ which is $1 / 3$ the wavelength at $200 \mathrm{MHz}$ ). Thus acceleration does not remain fixed at the crest of the rf waveform, but rather crosses over it one or more times. The nonscaling FFAG demonstrates at least two operational modes for rapid acceleration. These are discussed in the following. 
Near-crest regime. Cavity frequency and phases are selected to keep the beam as near crest as possible, reducing the total accelerating voltage and providing an almost constant acceleration rate. To first order, on-crest acceleration effects a simple translation of the energy coordinate; the pathlength differences cause a simple shearing of the phase space along the phase coordinate; both of which are linear effects. However, for large amplitude motions the cosine/parabolic nature of the waveform/pathlength becomes manifest and introduces phase space distortions. With proper optimization, this mode is the most conserving of longitudinal phase space, but acceleration is limited to afew turns else the machine acceptance is compromised. The number of turns (typically $\leq 10$ turns) is, in general, inversely proportional to frequency. This mode is optimal when the primary beam loss mechanism is due to a short decay lifetime, as is the case for muons, where only losses accruing in a few turns are acceptable. This mode appears to lessen distortion over a significant area of phase space when compared with other modes.

Cross-crest regime. An elaboration of this idea is to accept cavity frequency and phasing so that particles cross back-and-forth across the crest as the pathlength variations occur. Although more turns can be supported, the acceleration rate may show a strong modulation due to progressively larger excursions from crest. The number of turns is effectively limited by the performance of the machine; i.e. the degree to which the phase space becomes convoluted and the tolerance of longitudinal emittance blow-up. A limitation further exists in the rf, where more turns does not necessarily imply less rf voltage because the net phase slip progresses relative to the crest. For high frequency $(\approx 100-200$ $\mathrm{MHz}$ ) and high-Q cavities $\left(\mathrm{Q} \geq 10^{5}\right), 25$ turns, or less, is likely to correspond

to a practical limit after which the net accelerating voltage required per turn does not decrease.

\section{RF systems for rapid acceleration in a FFAG}

\subsection{Criteria}

The enormous momentum acceptance and dynamic aperture of nonscaling FFAGs implies beam remains circulating in the machine regardless of the net acceleration; i.e. beam is lost only from physical restrictions on machine orbits, and this occurs at energies outside of injection and extraction (for this case $<6 \mathrm{GeV}$ or $>20 \mathrm{GeV}$ ). The usual ideas of synchrotron longitudinal dynamics, such as RF bucket and synchronous phase, are not relevant to this type of machine, even when proper phase adjustments are possible. Consequently, in the context of rapid acceleration, the nondiscriminating performance of FFAGs implies a need to establish criteria by which to prescribe an rf system 
suited to a given application. The primary considerations appear to be: 1) total accelerating voltage; and 2) fidelity of the acceleration dynamics, or the degree of distortion which can be tolerated in the longitudinal phase space.

The present work investigates the simplest approach: the application and optimization of a single high-frequency, high-Q rf system to operate in the nearcrest regime; since this allows lower voltage and may furnish a less distorted phase space than cross-crest regime when an equal number of turns are employed. Modest elaborations, such as second harmonic or a mixing of several fundamental frequencies to produce a waveform that better matches the beam traversal times, are also considered.

\subsection{FFAG RF system}

The choice of radio-frequency is influenced by machines up and downstream. The upstream cooling channel leads us to consider 100 and $200 \mathrm{MHz}$ (ref??). The choice of shunt resistance $R$ and quality factor $Q$ is influenced by the RF power requirements imposed by $\mathrm{GeV}$-per-turn acceleration. Three options are considered: (i) low-Q $\left(<10^{3}\right)$; (ii) medium-Q $\left(\approx 10^{5}\right)$; (iii) high-Q $\left(>10^{7}\right)$. Because $R$ and $Q$ tend to scale with one another, these options correspond to high- medium- and low-power, respectively. The broadband option (i) allows the ideal re-phasing but has the disadvantage of comparatively low achievable acceleration voltage $(1 \mathrm{MeV} / \mathrm{m}$ or less).

Regarding options (ii) and (iii), there are CERN[13,14] designs available for $200 \mathrm{MHz}$ normal conducting (NC) and super conducting (SC) cavities, and for $400 \mathrm{MHz} \mathrm{SC}$ cavities that provide a starting point for extrapolation. A design with $R=14$ Mohm, $Q=7 \times 10^{4}$ and $2 \mathrm{MV}$ gap voltage is within reach of present NC technology and the peak rf power is some $250 \mathrm{MW}$ distributed between 1800 cavities. However, the filling time of $350 \mu \mathrm{s}$ is too long to allow rephasing. The modulation could be achieved by vector addition of a constant in-phase term and a varying quadrature term. Unfortunately, to sweep the phase correctly requires a quadrature modulation term comparable with the in-phase carrier. Thus the demands of the "quadrature-subsystem" are no different from the in-phase RF system. Given the large power requirement of NC cavities, a FFAG for rapid acceleration would clearly benefit from the adoption of superconducting cavities (SC) with quality factors ranging from $10^{7}$ to $10^{9}$. In either case, $\mathrm{NC}$ or $\mathrm{SC}$, pure sinusoid operation is the only mode possible. 


\subsection{RF waveform scenarios}

Ideal phases: Let us set aside the technological problems of on-crest operation. For a sinusoidal waveform, the ideal phases at cavity arrival times are zero since $\cos (0)=1$. One may then compute the ideal phases at the start of each turn so that the integrated phase at arrival is zero. The ideal phases turn-byturn for a single reference bunch are known; but one must also consider other bunches in the train. We assume that the RF system is not sufficiently agile to make adjustments for individual bunches, but rather that it runs at constant frequency during the train and that turn-by-turn phase adjustments can be made, for the purposes of simulation, in the beam gap. (Later, tiny lattice adjustments allowed the fixed-frequency of the cavities to exactly match the frequency of the bunch train.)

Best frequency and phases: The cavities are assumed to operate at a single frequency that we are free to optimize so that near-crest acceleration results. Also, one may set the initial individual cavity phases, consistent with that frequency, at the start of the first turn only.

The results from ideal phases will serve as a benchmark against which the more practical best phases scheme is to be compared. In addition to these two basic scenarios, a flat-top can be imposed on the RF waveform by the addition of the second harmonic so as to reduce the influence of phase variations.

\subsection{Optimization strategy for best frequency and phases}

There exist many possible optimization strategies. We report only that one which aims to give the reference bunch the maximum acceleration on each turn. The optimization is based on reference particles and, strictly speaking, it cannot be used directly to maximize input acceptance or minimize output emittance. However, extensive studies demonstrated that the acceptance and performance of the FFAG depend critically on combinations of initial cavity phasing, rf overvoltage, and the time scale set for acceleration, which are discussed below.

Cavity phasing: Initially, a gedanken is performed: the ideal arrival times $t_{i j}$ cavity-by-cavity (index $j=1, \ldots M$ ) and turn-by-turn (index $i=1, \ldots N$ ) are calculated and recorded for a single synchronous particle in the reference bunch assuming ideal energy gains. One makes an initial guess at the frequency $\omega$ and then calculates the phases $\phi_{i, j}=\omega t_{i j}$ at the gap-crossing times of the ideal particle. One then forms the square deviation of these phases from their average values, summed over all rf stations and turns: $S=\sum_{i=1}^{N} \sum_{j=1}^{M}\left(\phi_{i j}-\bar{\phi}_{j}\right)^{2}$ where $\bar{\phi}_{j}=\sum_{i}^{N} \phi_{i j} / N$. A search is then made to find the "best frequency" 
which minimizes $S$. If, at the start of the first turn, each cavity phase is set equal to the negative of the average value resulting from the optimization,

$-\bar{\phi}_{j}$, the phase at subsequent cavity arrival times will best approximate the ideal zero value. An improvement results if the lattice is adjusted slightly to make the best frequency equal to the frequency of the bunch train.

Over-voltages: Because every bunch arrives displaced from the ideal phase, the acceleration must be made tolerant of poor phasing. Since the time of Veskler[15] and McMillan[16], it has been recognized as advantageous to use an "over-voltage". One increases the voltage beyond the nominal accelerateon-crest value to compensate for arrivals which lag or lead the wave. Under the condition of rapid acceleration, "phase stability" is not guaranteed because of the path length variations. Nevertheless, a modest over-voltage is found to be of enormous benefit to the dynamical stability and performance of the machine, although stringent limits on the acceleration timescale remain. The over-voltage is chosen by a numerical optimization procedure so as to minimize the variation of the extraction energy and is based on tracking.

Acceleration Period: Acceleration near crest can only be sustained for a relatively small number of turns, inversely depending on frequency. As the number of turns increase so do the phase deviations from crest. Once the phase difference during acceleration exceeds $90^{\circ}$, there is a dramatic drop in the number of particles accelerated to extraction energy. This, in effect, sets the limits on the time scale for near-crest operation which, although limited in number of turns, has the advantage of a large machine acceptance that includes a low-distortion region of phase space of useful size.

\section{Simulations of longitudinal dynamics}

The simulation model assumes complete decoupling of the longitudinal from the transverse motion. The $6-20 \mathrm{GeV}$ ring, of $2.04 \mathrm{~km}$ circumference, is divided into 314 identical cells each with an RF station. Although the station could comprise up to 6 cavities, the energy gain is lumped in a single element. The particle arrival time depends on path length which is directly obtained from the circumference-change versus energy curve of figure 5. Initially, the longitudinal phase plane is uniformly flooded with trial particles, but with a momentum spread limited to $\pm 10 \%$. (The machine's energy acceptance extends from $90 \%$ of the injection energy to $110 \%$ of the extraction energy.) Particles which then accelerate to with $\pm 10 \%$ of the nominal extraction energy are considered to be within acceptable limits for extracted beam. The particles that survive these output cuts are recorded and used to map out the corresponding input admittance of the machine. Although a wide variety of cases has been considered and is reported elsewhere[17,18], here we present 
two indicative cases using 200 and $100 \mathrm{MHz}$ rf and describe the important trends in machine performance.

\section{$5.1200 \mathrm{MHz}$ acceleration}

To reduce electric field gradient one attempts to stretch near-crest acceleration over several turns. Although numerous cases were studied involving 5-10 turns, here we consider acceleration in five (5) turns of the FFAG which has been found to be, more or less, a practical limit.

Ideal phases: When the ideal turn-by-turn re-phasing, and the nominal RF voltage of $2.8 \mathrm{GV} /$ turn, is used an input admittance of $1.18 \mathrm{eV} . \mathrm{s}$ is successfully accelerated to $20 \mathrm{GeV}$ in 5 turns; see figures 6 and 7 . Notice, that despite the ideal phasing, the transport is clearly non-linear. If one adds second harmonic to the basic scheme, then the admittance rises to $2.12 \mathrm{eV}$.s.

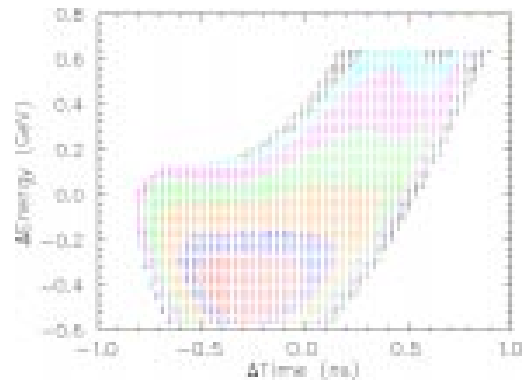

Fig. 6. $\pm 10 \%$ Band from input acceptance

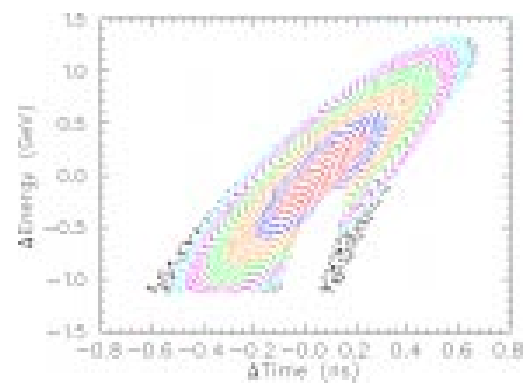

Fig. 7. Maps to the output emittance.

Best frequency and phases: If one uses a single frequency, and fixed initial cavity phases, then the desired acceleration is not achieved unless an overvoltage is employed. A $25 \%$ over-voltage yields a $1.5 \mathrm{eV}$.s admittance (Fig. 8). If one adds 2 nd harmonic (Fig. 9), then one needs $(4 / 3) \times 1.25$ of the nominal voltage, and the admittance rises to $2.28 \mathrm{eV}$.s. The input admittances are similar in shape to that in figure 6 except that their time-width is halved. Again, when considering the entire region, the transport is nonlinear, but the emittance which corresponds to the central region is reasonably well conserved.

It is interesting to consider whether, for fixed GV/turn, the number of accelerating stations has any influence upon beam quality. Using a $25 \%$ over-voltage and ranging from 100 to 600 stations, there is no systematic variation and the admittances span 1.2 to $1.5 \mathrm{eV}$.s.

Conclusion: For 5-turn acceleration, there is little difference in the output emittance between the use of "ideal phases" versus using a combination of "best phases" and an over-voltage. When second harmonic is employed, the useful acceptance is greater when the "best phases" scenario is adopted, and 


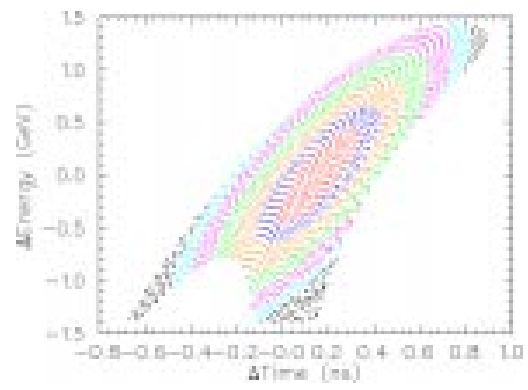

Fig. 8. Output emittance, single harmonic.

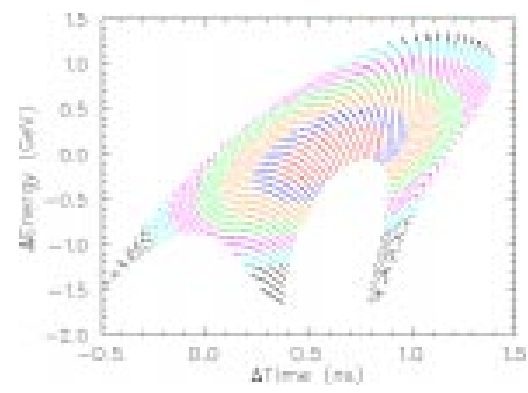

Fig. 9. Output emittance, dual harmonic.

is typically doubled. In all cases, the overall transport is non-linear with the useful, or preserved, phase space area comprising roughly one half of the full admittance. However, since most beams do not approach such large longitudinal emittances, nonlinear transport issues do not pose serious concerns with the operation of these machines in a rapid acceleration mode.

To first order, the phase slippage will increase linearly with time; and so one expects the transmission to fall in a roughly parabolic manner as the number of turns is increased. We have not achieved useful acceleration over more than five turns unless either (i) the ideal phases are used; or (ii) dual harmonic; or (iii) one allows each cavity (or groups of cavities) to run at its own individually optimized frequency. In the former case, up to 10-turn; and in the latter cases 7-turn and 6-turn, respectively, acceleration is possible. However, when there are many different cavity frequencies, the task of re-setting the first-turn phasing is a daunting task, particularly if SC cavities with a typical $1 \mathrm{~s}$ filling time are adopted.

\subsection{Acceleration with $100 \mathrm{MHz} R \mathrm{RF}$}

Since the phase slips accumulate half as quickly when the RF is halved, one anticipates that $100 \mathrm{MHz}$ acceleration will be less compromised by using a larger number of turns. Based on the results of the $200 \mathrm{MHz}$ case studies, we have considered only the use of a "best frequency and phases" for $100 \mathrm{MHz}$ rf. Certain general trends can be noted. The input admittance rises almost linearly from 0.2 to $4.1 \mathrm{eV}$.s as the number of turns is reduced from fourteen to six and the voltage-per-turn is raised from 1.25 to $2.92 \mathrm{GV}$. For example, 10turn acceleration with a modest $25 \%$ over-voltage super-posed on the nominal voltage of $1.4 \mathrm{GV} /$ turn, yields a $2.3 \mathrm{eV}$.s acceptance; see figures 10 and 11 . If one adds second harmonic, the admittance rises to $4.2 \mathrm{eV}$.s. Varying the number of rf stations from 100 to 600 again has little influence; admittances vary from 2.22 to $2.33 \mathrm{eV}$.s. 


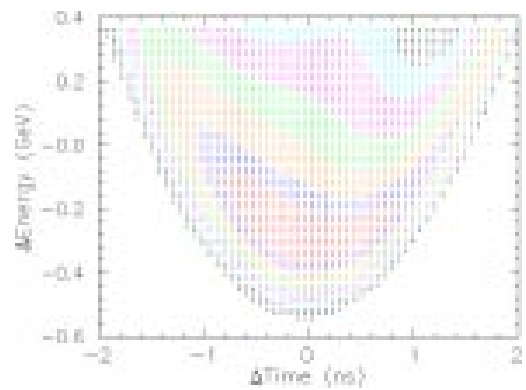

Fig. 10. $\pm 10 \%$ Band from input acceptance.

Conclusion: For acceleration with $100 \mathrm{MHz}$ RF using a single frequency and fixed "best phases", the optimum admittance of the machine is achieved when the number of turns appears to be eight or nine combined with modest overvoltages. However, admittances comparable to the $200 \mathrm{MHz}$ case can be achieved in 10,11 or even 12 turns with a corresponding and advantageous decrease in rf gradient. For $100 \mathrm{MHz}$ rf, use of the second harmonic in combination with an overvoltage and best phasing produces a tremendous longitudinal acceptance in a FFAG for a rapid-acceleration application.

\section{Summary}

Nonscaling FFAGs have a strong advantage in applications requiring rapid acceleration of large-emittance beams by providing a transverse admittance beyond conventional scaling FFAGs. The magnet layout (horizontally-focussing quadrupole and horizontally-defocussing combined-function magnet) with appropriate optimization represents an innovation in FFAG lattice design, and has been described in detail in earlier publications. This design approach produces the most efficient and compact linear machine design in terms of circumference and magnet aperture. (Circumference reduction approaches a factor of two from scaling FFAG designs developed for the same application.) Magnet aperture could be further circumscribed by the adding strong nonlinear field components, in particular sextupole and octupole, but at great expense to the transverse dynamic aperture and linear transport. The slowly-changing optics which characterize this nonscaling FFAG design have an additional advantage in the suppression of nonlinear resonances for rapid, or moderately-rapid acceleration applications.

At least initially, it was felt that the circumference change, or phase-slip, posed a serious problem, but since then numerous solutions have been proposed. However, most of the previous solutions had the disadvantage of requiring impractical rf power by applying very low-frequency rf, or broadband rf to accomodate the phase-slip. This work represents the first successful study 
of the application of reasonably high-frequency and high-Q cavities to rapid acceleration in a fixed-field accelerator, thereby dramatically reducing the $\mathrm{rf}$ power required in previous solutions.

Rapid acceleration with no adjustments possible in the cavity phasing, however, show strict operational constraints in order to meet stablility requirements in the output phase space and energy distribution. For near-crest operation, which displays stable performance across a large longitudinal machine acceptance three factors were found to be critical and interrelated: optimal cavity frequency and starting phases, overvoltages, and the timescale for acceleration (the total number of turns in the machine). Only a few turns are supportable in the context of a large and practically stable machine admittance and, even then, it is only achieved by applying different initial phases on rf stations and allowing modest overvoltages (typically 20-40\%). Even though little phase-space increase is evident in the final emittance, the transport, which tends to increase the momentum spread and decrease the bunch length, is still nonconventional,

Specifically, in the application of 200 and $100 \mathrm{MHz}$ rf, the number of turns increases from 5 to 10 , respectively, indicating the expected general dependence on the inverse of the frequency. In this approach, a hard limit occurs when the phase-slip exceeds $90^{\circ}$ from crest at which point a sharp falloff of stable and useful transmission occurs. (One can force an increase in the number of turns, but at great expense in the overvoltage and at the cost of even greater convolution of the phase space.) Applying a second harmonic has a dramatic effect on the transmitted phase space area, but had only a minor impact on the number of turns.

In practical terms, when $200 \mathrm{MHz}$ RF is utilized, useful admittances ( $\geq 1 \mathrm{eV} . \mathrm{s})$ can be achieved with acceleration in 6 or less turns using either either a phaseagile or a high-Q fixed-frequency RF system and a modest over-voltage in a FFAG. Surprisingly, there appears to be little advantage in using a phase-agile or low-Q cavity to the overall performance when operating in the on- or nearcrest regime. For the case of $100 \mathrm{MHz}$ RF, study of a fixed frequency system, shows that a useful output emittance of $(\geq 2 \mathrm{eV} . \mathrm{s})$ is realized for acceleration in 10 or less turns. In all cases, addition of second harmonic roughly doubles the phase space conserved within the defined cuts.

In conclusion, the nonscaling FFAG coupled with the rf approach developed here presents not only a solution to rapid acceleration, but also a new acceleration technique. Ultra-large emittances are successfully transported in a conventional machine with minimal complexity in components (when compared with the more design-intense magnets required for a scaling FFAG). The use of only linear fields form the basis for the tremendous transverse and longitudinal acceptance, one that is large in comparison with conventional 
acceleration, including traditional FFAG machines. With appropriate rf technique and design, this large acceptance was found to be preserved despite the problems introduced by phase-slippage. Specifically, this approach provides the necessary transverse and longitudinal acceptance match to high-energy muon beams with little or minimal cooling in place. Based on this work, it looks promising to build a chain of muon accelerators from FFAGs and replace the costly and somewhat restrictive RLAs, which so far have been the baseline accelerator for the feasibility studies of a Neutrino Factory in the U.S $[5,6]$.

\section{References}

[1] D. Kerst, K. Symon, L. Laslett, L. Jones, K. Terwilliger, "Fixed Field Alternating Gradient Particle Accelerators", pgs. 32-35, Proc. of CERN Symposium on High Energy Accelerators, Geneva, June 11-23, 1956.

[2] C. Johnstone and S. Koscielniak, "Recent Progress on FFAGs for Rapid Acceleration," Proc. of the APS Snowmass 2001, The Future of Particle Physics, Snowmass, CO, June 28-July 21, 2001. C. Johnstone, "Recent Studies of FFAGs in the USA", Proc. of the 16th International Conference on Cyclotrons and their Applications, May, 2001, available at http://meetings.nscl.msu.edu//cyclotrons2001 C. Johnstone, W. Wan, and A. Garren, "Fixed Field Circular Acceleration Design", Proceedings of the 1999 Particle Accelerator Conference, New York, NY, Mar. 29-Apr. 2, 1999, pp. 3068.

[3] F. Mills, "Linear Orbit Recirculators", pgs. 693-696, Proc. of the 4th International Conference on the Physics Potential and Development of the $\mu+\mu-$ Colliders", San Francisco, CA, Dec. 10-12, 1997. C. Johnstone, "FFAG design", talk, pgs. 696-698, Proc. of the 4th International Conference on the Physics Potential and Development of the $\mu+\mu$-Colliders", San Francisco, CA, Dec. 10-12, 1997.

[4] The $\mu+\mu-$ Collaboration, " $\mu+\mu-$ Collider: A Feasibility Study", BNL-52503, Fermi-Conf-96-/092, LBNL-38946, July 1996.

[5] N. Holtkamp and D. Finley, eds., A Feasibility Study of a Neutrino Source Based on a Muon Storage Ring, Fermilab-Pub-00/108-E (2000).

[6] S. Ozaki, R. Palmer, M. Zisman, and J. Gallardo, eds., "Feasibility StudyII of a Muon-based Neutrino Source", BNL-52623, June, 2001, available at http://www.cap.bnl.gov/mumu/studii/FS2-report.html.

[7] C. Johnstone, D. Neuffer, and E. Forest in "The CERN Neutrino Factory Study", Chapter 6 (the $2-10 \mathrm{GeV}$ recirculator design), available at cdj.home.cern.ch/cdj/yrep/yr.htm C. Johnstone, D. Neuffer, E. Forest, "A 3$11 \mathrm{GeV}$ Recirculating Linac for Muon Acceleration: Design and Simulation" Proc. of the 2001 Particle Accelerator Conference, Chicago, IL, June 18-22, 2001. 
[8] K. Symon, MURA-KRS-6, (MURA-43) (1954); K. Simon, et al, Phys. ReV. 103 p. 1837, (1956)..

[9] S. Machida, et al, "Beam Optics Design of an FFAG Synchrotron", (MOP1B20), submitted to EPAC 2000, and Y. Sato, et al, "Development of a FFAG Proton Synchrotron", (MOP1B21), submitted to EPAC 2000.

[10] W. Wan, unpublished, 1998.

[11] FFAG02, Tsukuba, Japan, Feb., 2002, http://hadron.kek.jp/FFAG/ffag02 FFAG Workshop, CERN, Geneva, Switzerland, July 3-4, 2000. FFAG00, KEK, Tsukuba, Japan, Oct. 11-13, 2000.

[12] A. Garren, presented at the CERN FFAG Workshop, Geneva, Switzerland, July3-4, 2000.

[13] D. Boussard et al: "Design Considerations for the LHC $200 \mathrm{MHz}$ RF System", LHC Project Report 368, January 2000.

[14] E. Chiaveri \& R. Losito: private communication.

[15] V.I. Veskler: Comptes Rendus de l'Academie des Sciences URSS 43, No. 8, pgs. 329-331 (1944) and 44, No. 9, pgs.365-368 (1944).

[16] E.M. NcMillan: Phys. Rev., 68, pg. 143 (1945) and 69, pg. 534 (1946).

[17] S. Koscielniak: TRIUMF design note TRI-DN-01-15, September 2001.

[18] S. Koscielniak: TRIUMF design note TRI-DN-02-06, April 2002. 